\title{
Efectos distributivos de los tributos ecológicos. Apuntes metodológicos
}

\author{
TOMÁs J. LÓPEZ-GUZMÁN GUZMÁN \\ FERNANDO LARA DE VICENTE*
}

\begin{abstract}
The aim of this paper is to propose a methodology that allows us to achieve a correct design of an ecological tax avoiding the fact that certain groups turn out to be more affected by the introduction of an eco-tax. The methodology guidelines are centred on the analysis of the basic structure of an environmental toll, as well as determining reference groups and the analysis of distribution effects. The main result obtained is that the introduction of environmental taxes must take into account the negative impact on certain groups, above all when electric energy is levied. Thus, we conclude that it would be essential that before introducing ecological taxes, the necessary studies were carried out in order to avoid the negative impact; or alternatively, that policies of compensation and mitigation were included in the appropriate regulations.
\end{abstract}

Keywords: ecological taxes, methodology, distribution effects, fiscal incidence, European Union, Mexico.

\section{Resumen}

El objetivo de este trabajo es presentar una propuesta metodológica para realizar un correcto diseño de un tributo ecológico que evite que determinados grupos sean los más perjudicados con la introducción de una ecotasa. Las pautas metodológicas se centran en el análisis de la estructura básica de un tributo ambiental, la determinación de los grupos de referencia y el análisis de los efectos distributivos. El principal resultado es que la introducción de los tributos ambientales debe tener en cuenta el probable impacto negativo en determinados grupos, sobre todo cuando se grave la energía eléctrica. Por tanto, sería necesario que previo a la introducción de los tributos ecológicos se elaborasen estudios necesarios para evitar su impacto negativo o, en su caso, se abordasen en la misma regulación políticas de compensación y mitigación.

Palabras clave: tributos ecológicos, metodología, efectos distributivos, incidencia fiscal, Unión Europea, México.

*Universidad de Córdoba. Correos-e: tomas.lopez@uco.es y lara.fernando@uco.es. 


\section{Introducción}

El ambiente se ha convertido en los últimos años en uno de los elementos clave de las diferentes ciencias, entre ellas la económica. La necesidad de proteger el entorno natural en la realización de los diversos procesos productivos ha llevado a la ciencia económica a diseñar variados instrumentos para incluir el ambiente en la fijación de los costes. Fruto de ellos son los llamados instrumentos económicos para la protección del medio, que persiguen la aplicación de metodologías, modelos y teorías ya existentes a este nuevo factor ambiental. Entre estos instrumentos destacamos los permisos de emisión negociables, presentes en algunos países y que, gracias al Protocolo de Kyoto, también se han establecido en la Unión Europea. Asimismo, en Europa han venido aplicándose desde hace ya algunos lustros los tributos ecológicos, que permiten interiorizar los efectos externos provocados en el ambiente. La generalización en los sistemas fiscales de esta suerte de tributos está llevando a la consideración de un análisis en profundidad de los efectos que producen tanto sobre el entorno natural como sobre el propio sistema tributario. Además, recordemos que estos tributos se basan en el principio de "quien contamina paga". Sin embargo, pensamos que en el diseño de estos tributos, junto con otros elementos de carácter ambiental, también es necesario (e imprescindible) tener en cuenta quién realmente paga el tributo y, sobre todo, determinar los efectos distributivos que estos tributos ocasionan, ya que podría ocurrir, paradójicamente, que se lograse la protección del medio pero con cargo a las familias con rentas más bajas.

El análisis de los efectos distributivos de los tributos ecológicos se convierte, por tanto, en un elemento clave en el diseño de la ecotasa, y de su correcto diseño dependería, en principio, el desarrollo del tributo. Así, se han establecido ya diferentes metodologías -por todos- (OCDE, 1994) para emprender el estudio de los efectos distributivos de los tributos ecológicos en general. En este trabajo presentamos una propuesta metodológica para analizar los efectos distributivos que los tributos ecológicos que gravan la contaminación atmosférica causan sobre las familias, a partir de dos hechos contaminantes que, en nuestra opinión, se han configurado como clave en el desarrollo de estas ecotasas: la producción de energía eléctrica utilizando fuentes contaminantes, y el consumo de carburantes en elementos de transporte. 
En el primer apartado de este trabajo proponemos cómo debería ser el diseño del tributo ecológico y, concretamente, el de aquellos tributos destinados a luchar contra la contaminación atmosférica. En el segundo apartado presentamos los principales grupos de referencia que la literatura analizada elige para realizar ese estudio. En el tercer apartado analizamos los impactos distributivos de un tributo ecológico que grave las emisiones contaminantes a la atmósfera, y dividimos dicho análisis en tres aspectos: los efectos directos del tributo, sus efectos indirectos y el destino de la recaudación que de él se obtiene. En el cuarto apartado nos aproximamos al estudio de la incidencia fiscal de la ecotasa, es decir, quién debería pagar el impuesto y quién realmente lo paga. Asimismo, no debemos olvidar que si el tributo ecológico presentase efectos negativos sobre determinados grupos de referencia, sería necesario aplicar políticas de compensación o mitigación.

\section{Estructura básica de un tributo ambiental}

La estructura de cualquier tributo ambiental se define atendiéndose al producto, gas o sustancia contaminante objeto de gravamen; en nuestro caso, la contaminación atmosférica. En conformidad con Poterba (1991: 73-75), el análisis de la estructura de un tributo ambiental para la contaminación atmosférica debería centrarse en cinco elementos clave: el sujeto pasivo, el hecho imponible gravado, la determinación de la base imponible, la fijación de la tarifa y la cuantificación de la cuota íntegra. A continuación describimos cada uno de los elementos comentados, en referencia al tipo de tributo ambiental elegido para lograr la reducción de la contaminación atmosférica.

\section{a) Sujeto pasivo}

El sujeto pasivo de un tributo ecológico para el control de la contaminación atmosférica debe ser aquella persona, física o jurídica, que realiza la actividad degradante del medio natural. El sujeto contaminante se identificaría, pues, con alguno de los siguientes agentes (Borrero Moro, 1999: 129): productor de actividades contaminantes, consumidor de productos contaminantes, o titular de los bienes patrimoniales con los que se realiza la actividad contaminante. 
A la vista de estos tres supuestos, el sujeto pasivo del tributo ambiental diferiría si éste gravase la producción de energía eléctrica generada por fuentes contaminantes o el consumo de combustible por el automóvil. En el primer caso, el sujeto pasivo sería la empresa productora de energía eléctrica, mientras que en el segundo lo sería el consumidor del carburante. No obstante, en este segundo caso, dada la dificultad, si no imposibilidad, para la administración tributaria de definir al consumidor como sujeto pasivo, podría adoptarse como solución exigir el gravamen o bien al fabricante, o bien al importador del carburante, y posteriormente arbitrarse las medidas necesarias para que el tributo pudiera repercutirse sobre el consumidor, es decir, el realizador de la actividad degradante del medio natural.

\section{b) Hecho imponible}

Constituyen el hecho imponible de esta clase de tributos ambientales las emisiones directas a la atmósfera o el consumo de un producto que ocasione, directa o indirectamente, la contaminación atmosférica. No obstante, la literatura disponible ofrece, sobre este particular, un doble punto de vista. Desde la óptica de los efectos o beneficios estrictamente ambientales, la doctrina parece abogar claramente por la imposición directa de las emisiones ocasionadas por las fuentes contaminantes; sin embargo, al considerar el posible coste administrativo de los mecanismos o procedimientos de control de las emisiones generadoras del hecho imponible, son múltiples los autores que consideran que, por un aspecto exclusivamente de eficiencia, puede resultar conveniente gravar el consumo -final o intermedio- del producto contaminante, e introducirse, en su caso, deducciones para los procesos de descontaminación (véase: Hamilton y Cameron, 1994; Whalley y Wigle, 1991; Poterba, 1991).

\section{c) Base imponible}

La determinación de la base imponible es una tarea exclusivamente de carácter técnico, al constituirse por unidades físicas bien del producto, bien de las emisiones. Su determinación se realizaría aplicando métodos de estimación directa. Adicionalmente, cuando no sea posible la aplicación de la estimación di- 
recta en determinadas regulaciones legales, ${ }^{1}$ se establecen dos procedimientos secundarios para la determinación de la base imponible: de una parte, un procedimiento de estimación objetiva de la base, y, de otra, uno de estimación indirecta basada en cálculos o estimaciones preexistentes en poder de la administración tributaria.

\section{d) Tarifa}

La fijación de la tarifa del tributo es uno de los aspectos más complejos del diseño de un tributo ambiental, al punto de que la disyuntiva entre la utilización de un tipo de gravamen único o uno con varios tramos ha sido objeto de un profundo debate doctrinal. Como procedimientos opcionales para la fijación del tipo de gravamen, ciertos autores se han mostrado partidarios de predefinir el objetivo ambiental -por ejemplo, conseguir los objetivos de la Cumbre de Río (Cornwell y Creedy, 1996)- estimando a posteriori el tipo de gravamen preciso para su logro; otros, en cambio, recurren a métodos econométricos -entre ellos, Hamilton y Cameron (1994: 388)- para la determinación de la tarifa, y, como tercera alternativa, algunos han optado por utilizar tarifas correspondientes a tributos ambientales vigentes o propuestos -tal es el caso de López-Guzmán Guzmán (2002) respecto a la tarifa de una ecotasa aplicada en España.

\section{e) Cuota integra}

La cuota íntegra, expresada en unidades monetarias, se obtendría aplicando a la base imponible el tipo de gravamen establecido. Asimismo, si se autorizase algún tipo de deducción en la cuota íntegra del tributo ecológico (para incentivar, por ejemplo, la inversión en procesos más respetuosos con el medio natural), se obtendría la cuota líquida.

De esa cuota íntegra dos son los aspectos concretos que han de analizarse para la determinación de los efectos distributivos de la tributación ambiental: su posible traslación a los consumidores finales y, por otro lado, qué porción o parte de ella podría afectar directamente a los grupos de referencia -lo que hemos llamado efectos directos-, y cuál podría afectarles indirectamente -lo que se denomina efectos indirectos.

\footnotetext{
${ }^{1}$ Entre otros, el impuesto francés sobre la contaminación atmosférica y el impuesto de la Comunidad Autónoma de Galicia (España) sobre la contaminación atmosférica.
} 
En relación con el primer aspecto, la Organización para la Cooperación y el Desarrollo Económicos (OCDE, 1994: 57-58) señala que la estimación de los efectos distributivos de un tributo ambiental ha de realizarse sobre la base de una triple hipótesis de traslación: la traslación completa de la cuota íntegra (hipótesis avanzada), la traslación parcial de la cuota (hipótesis intermedia) y una traslación nula (hipótesis conservadora). La consideración de estas tres hipótesis mostraría cómo diferirían los resultados obtenidos en el análisis, dado que, en el primer caso, la traslación total, los efectos distributivos únicamente se manifestarían en los consumidores finales, mientras que en las dos restantes hipótesis, el impuesto también afectaría a factores de producción: principalmente los accionistas y los trabajadores de las empresas sometidas a tributación y los propietarios de los recursos naturales gravados.

El segundo aspecto de la cuota por analizar consistiría, como ya se ha comentado, en determinar la parte de la cuota íntegra que afectaría directamente al grupo de referencia y aquella que lo haría de forma indirecta. La literatura disponible sobre la materia considera que un tributo ecológico para el control de la contaminación atmosférica afectaría de forma directa a los consumidores finales por la vía del consumo de dos tipos de productos: la energía doméstica y el carburante para el automóvil. Consiguientemente, en este tipo de análisis habrá de procederse a la estimación del porcentaje del gasto que correspondería a cada uno de estos consumos para los diferentes grupos de referencia analizados. Al mismo tiempo, es claro que el consumo de bienes y servicios en cuya producción se hubieran empleado inputs sometidos al impuesto ecológico implicaría efectos indirectos del impuesto en el grupo de referencia considerado en el análisis.

\section{Definición de los grupos de referencia respecto del impacto distributivo}

La determinación de los grupos de referencia, es decir, los grupos respecto a los que se realizará la valoración de los efectos distributivos del tributo ambiental, es, sin duda, uno de los aspectos centrales en esta suerte de análisis. La literatura fiscal ambiental (véanse, entre otros, Poterba 1991; Smith, 1992; Hamilton y Cameron, 1994; Symons, Proops y Gay, 1994; Cornwe11 y Creedy, 1996) presenta, en todo caso, un claro predominio por la utilización de los niveles de renta-expresados en deciles, 
cuartiles o quintiles- como grupo de referencia para el estudio de los efectos distributivos de la imposición ambiental, desde la perspectiva tanto del gasto como de los ingresos. Symons, Proops y Gay (1994), por ejemplo, utilizan en su investigación los grupos de renta referidos a hogares del Reino Unido, mientras que Hamilton y Cameron (1994) refieren su estudio a los hogares canadienses clasificados por niveles de renta.

Por su parte, Whalley y Wigle (1991) realizan su estudio sobre los efectos distributivos de la imposición ambiental tomando como referencia distintas áreas geográficas o grupos regionales: la Unión Europea, Norteamérica (Estados Unidos y Canadá), Japón, otros países de la OCDE, países exportadores de petróleo y otros (incluida Rusia). Llevan a cabo la delimitación de estas regiones atendiendo al nivel de consumo y producción, con lo que promueven el debate de si el tributo ambiental debería aplicarse a la producción o al consumo.

La estimación de los efectos distributivos de la tributación ambiental respecto a un solo grupo de referencia dista de ser, no obstante, una pauta generalizada en esta clase de análisis. Al contrario, son muchos los autores que han optado por la realización de estudios empíricos referidos simultáneamente a varios grupos de referencia y obtienen, de esta manera, una visión más completa de cuáles son los ciudadanos que realmente ganan y pierden con la introducción de un gravamen ambiental. Johnson, McKay y Smith (1990), entre otros, refieren su trabajo sobre los efectos distributivos de un tributo ecológico, aplicado al consumo de combustible, a tres grupos de referencia diferentes: las regiones, los deciles de renta y el número de miembros en la familia. En lo que concierne a este último grupo de referencia, Brännlund y Kriström (1997) apuntan, en cualquier caso, que para efectos analíticos conviene proceder a una nueva desagregación conforme a las características cualitativas de los miembros de la familia: adultos que trabajan, niños o jubilados.

\section{Análisis de los impactos distributivos de un tributo ecológico}

Tras la estimación de la cuota íntegra del tributo ecológico, expresada, como se ha señalado, en unidades monetarias, a continuación se asigna el incremento fiscal a cada uno de los grupos anteriormente definidos. Esta distribución de la carga impositiva debería realizarse atendiendo a dos clases de demanda: la de- 
manda final, o consumo directo del producto gravado por parte de los grupos de referencia, y la demanda intermedia, o consumo o utilización en la producción de otros bienes y servicios. Los efectos distributivos de un tributo para el control de la contaminación atmosférica se obtendrían, por tanto, como resultado de la interrelación de tres factores diferentes (Pearson, 1995): el consumo directo de determinados bienes, los inputs de energía utilizados en los procesos de producción y el destino de los ingresos obtenidos mediante el tributo ecológico.

La metodología utilizada en la designación de los efectos impositivos sobre el consumo directo de determinados bienes y el consumo de inputs por parte de las empresas contaminantes se atiene a pautas distintas y perfectamente diferenciadas (Symons, Speck y Proops, 2004: 12). En cuanto al efecto directo de un tributo ambiental, éste se determinaría mediante un análisis de microsimulación que emplea los modelos de consumo de los países -o áreas geográficas- estudiados para diferentes grupos de referencia, construidos sobre la base de los datos estadísticos facilitados por las encuestas de gastos familiares. El efecto indirecto del gravamen se valuaría, en cambio, a partir de un análisis input-output, trasladando los inputs de los procesos de producción a cada uno de los grupos de referencia que corresponde a las encuestas de gastos familiares. Asimismo, mediante un modelo de microsimulación se establecería la respuesta de consumo de los hogares ante un cambio en los precios de los bienes originados por los tributos ecológicos.

Más en concreto, y siguiendo a Symons, Proops y Gay (1994) y Cornwell y Creedy (1996), las etapas que deberían seguirse en la determinación de los efectos distributivos de un tributo ecológico para el control de la contaminación atmosférica serían las siguientes: primera, aplicación del modelo de microsimulación para estimar el impacto directo del tributo ambiental sobre el consumo de energía doméstica y de carburante para el automóvil realizado por los hogares, sobre la base de los datos facilitados por las encuestas de gasto nacionales; segunda, utilización de las tablas input-output para valuar el impacto indirecto de los tributos ambientales en los grupos de referencia, como consecuencia del incremento de los precios de los productos fabricados con materiales sujetos al impuesto; tercera, en las dos primeras etapas sería necesario plantear las tres alternativas anteriormente señaladas respecto a la traslación del tributo avanzada, intermedia y conservadora- a los consumidores fina- 
les; cuarta, cambios inducidos por el tributo ambiental en la demanda de los productos consumidos, con el apoyo del IFS simulation program for indirect taxation (SPIT); ${ }^{2}$ quinta, distribución de los pagos impositivos entre los diferentes intervalos o niveles del grupo de referencia utilizados, con la expresión de los resultados tanto en unidades monetarias como en porcentajes; y sexta, definición y exposición de las posibles políticas de compensación y mitigación aplicables a los niveles del grupo de referencia que pudieran resultar perjudicados por el tributo ambiental.

\subsection{Efectos directos derivados de la introducción de un tributo ecológico}

Para determinar los efectos directos de un tributo para el control de la contaminación atmosférica, deben especificarse, en primer lugar, cuáles serían los bienes afectados. De acuerdo con Pearson (1995), la aplicación de esta clase de tributos afectaría directamente a dos clases de gastos del grupo de referencia: el consumo de energía doméstica y el consumo de combustible para el automóvil.

Tras la especificación de estos dos grupos de gastos ha de procederse, en segundo lugar, a la delimitación del patrón o modelo de consumo actual de cada uno de los grupos de referencia. Si el grupo de referencia del estudio viniese constituido por los niveles de renta, dicho modelo se articularía en torno a la información disponible sobre el gasto realizado en estos dos bienes por deciles de ingresos y deciles de gastos de los hogares. Para obtener esta información ha de acudirse a fuentes estadísticas sobre las pautas de consumo de los ciudadanos. La Encuesta de Presupuestos Familiares proporciona estos datos en España; en Australia, la Household Expenditure Survey; en Estados Unidos, la Consumer Expenditure Survey; en el Reino Unido, la Family Expenditure Survey, y, por último, para el estudio comparado en diversos países de la Unión Europea, Eurostat.

Una vez estimado el gasto realizado por niveles de gastos deciles, quintiles o cuartiles-, tanto en valores absolutos (unidades monetarias) como en valores relativos (porcentajes), así como el porcentaje de consumo de cada bien correspondiente a cada nivel de gasto, la tercera fase del estudio se desglosaría en un doble cálculo: primero, el del cambio que induciría el impuesto

${ }^{2}$ La metodología para la aplicación de este programa de simulación puede encontrarse en Baker, McKay y Symons, 1990. 
ambiental en los hogares. Este análisis suele realizarse recurriendo a modelos econométricos y, más concretamente, al programa SPIT -tal como hacen, entre otros, Johnson, McKay y Smith (1990) y Symons, Proops y Gay (1994)-; segundo, la cuantificación de la cuota íntegra correspondiente a la demanda final de cada uno de los grupos de referencia.

Respecto a los resultados obtenidos por los estudios empíricos consultados sobre los efectos distributivos de un tributo ecológico sobre el consumo de energía doméstica, referido exclusivamente a la demanda final, todos concluyen que el gravamen, en mayor o menor grado, devendría regresivo. Tal regresividad se debería al impacto que el gravamen provocaría en el precio de la energía doméstica, y se explicaría por el hecho de que los hogares con rentas más bajas destinan un porcentaje más elevado de su gasto al consumo de este ítem que los hogares con rentas más elevadas. Sin embargo, en lo que se refiere al consumo de combustible para el automóvil, los resultados de esos estudios difieren entre sí, incluso hasta el punto de que algunos de ellos estiman una cierta progresividad del impuesto ecológico. La razón parece residir en el hecho de que los hogares con ingresos superiores, además de utilizar con relativa mayor frecuencia medios de transporte privados, cuentan con automóviles más potentes y, en consecuencia, son también mayores consumidores de combustible.

\subsection{Efectos indirectos de un tributo ecológico}

El segundo elemento de estudio en el análisis del impacto distributivo de un tributo ecológico es el camino recorrido por la demanda intermedia, por la vía de lo que hemos denominado efectos indirectos. Puesto que el consumo de inputs, causante de la contaminación atmosférica, lo realizan las empresas en proceso de fabricación de los productos, un porcentaje de la cuota tributaria -expresada en unidades monetarias- no incidirá de forma directa sobre los grupos de referencia. Las empresas contaminantes pueden, ciertamente, trasladar el tributo mediante un incremento de los precios pagados por los consumidores finales, con ello generan efectos distributivos en los grupos de referencia. Pearson (1995) considera, no obstante, que el análisis de estos efectos distributivos no sólo debería incluir a los consumidores finales sino, además, a los propietarios de los factores de producción (capital, trabajo y recursos naturales). 
En este segundo planteamiento, la literatura disponible propone como instrumento analítico la utilización de las tablas inputoutput. Con el concurso de estas tablas puede determinarse qué tipo de industria consume el producto contaminante o causa la emisión objeto de gravamen -la denominada demanda intermedia-. Una vez delimitados los sectores industriales y su demanda a través de los grupos que figuran en las tablas input-output, debe establecerse a continuación la correlación existente entre dichas tablas y las fuentes estadísticas que informan sobre el gasto del grupo de referencia; de esta forma se acotan los grupos de gasto a los que se trasladaría la cuota tributaria del impuesto ambiental.

Los autores consultados precisan, sin embargo, que debido a la complejidad de esta hipótesis, sería difícil determinar de una manera clara cómo la demanda intermedia se traslada a los consumidores finales de cada grupo de referencia. Esa complejidad parece tener su origen en la necesidad de realizar una doble simulación: por un lado, tras la concreción de la demanda intermedia, proceder al reparto de la carga con arreglo al consumo realizado por los diferentes sectores, y, por otro, establecer la correspondencia entre las tablas input-output y las fuentes estadísticas sobre los gastos de cada grupo de referencia. La complejidad de esta tarea radica, entre otras razones, en la falta de coincidencia entre los grupos. La utilización, a estos efectos, de las tablas input-output deviene una cuestión problemática por diversos motivos: ante todo porque los precios pagados por el combustible varían considerablemente entre los diferentes sectores; luego porque las tablas inputoutput no distinguen entre el uso energético y el no energético de los combustibles fósiles y, finalmente, porque ciertos combustibles fósiles constituyen en realidad recursos intermedios en la producción de otros bienes (Hamilton y Cameron, 1994: 390). Pearson (1995), por su parte, juzga necesario recurrir a las tablas input-output para calcular el cambio en el precio del producto final inducido por el impuesto y, en consecuencia, utiliza también un modelo de equilibrio general basado en la demanda de consumo y en la sustitución de los factores productivos. De ahí, a su vez, que sostenga que, para no incurrir en posibles errores, se harían necesarias demasiadas estimaciones de comportamientos de demanda y de parámetros técnicos.

Finalmente, la literatura analizada (por todos, según Smith, 1992: 8) señala otro elemento de dificultad en el estudio de los efectos indirectos. Así, tal contratiempo parte de que se considera que la traslación de un tributo ambiental correspondiente a la 
demanda intermedia estaría condicionada por cuatro elementos: el grado de monopolio, la competencia internacional, el grado de sustitución de los diferentes factores de producción y la velocidad de ajuste.

La conclusión que podemos extraer de los efectos distributivos indirectos de un tributo ambiental, analizados en los estudios empíricos que presentamos, es triple: primera, la dificultad para determinar el impacto provocado por la demanda intermedia; segunda, el impuesto sería más regresivo cuanto más afecte la introducción de un tributo ecológico a las industrias fabricantes de productos de primera necesidad -por ejemplo la industria alimenticia-; y tercera, en las hipótesis presentadas por los diferentes autores, el grupo de gasto más afectado es, evidentemente, el que corresponde a la energía y al combustible, con lo cual los efectos indirectos apoyan claramente los resultados derivados de los impactos distributivos de los efectos directos anteriormente calculados.

\subsection{Destino de la recaudación obtenida por la aplicación de tributos ecológicos}

Los efectos distributivos de un tributo ecológico para el control de la contaminación atmosférica se determinan no sólo reparando en los efectos directos e indirectos, sino también en el destino que se le dé a los ingresos obtenidos con él. La literatura sobre la materia considera que uno de los elementos clave para el éxito de esta clase de impuestos es que la recaudación del tributo ambiental sea lo suficientemente elevada como para incentivar la protección del medio y, además, conseguir el objetivo del doble dividendo. Como ejemplo de un tributo altamente productivo en términos recaudatorios, en el cuadro 1 se exponen los datos presentados en el estudio empírico realizado por Poterba en 1991. La recaudación expuesta en dicho cuadro procede de un tributo ecológico de 100 dólares por tonelada de carbono aplicado en diferentes países y áreas geográficas, y se expresa tanto en unidades monetarias como en porcentaje del producto nacional bruto (PNB).

De acuerdo con los expertos en fiscalidad ambiental, una elevada recaudación de esta suerte de tributación permitiría a las diferentes administraciones públicas no sólo arbitrar medidas para conseguir una mejora en la protección del medio natural, sino además contribuir al logro de objetivos macroeconómicos. En contraposición, sin embargo, no debe ignorarse que cuanto ma- 


\section{Cuadro 1}

Ingresos obtenidos mediante un tributo sobre el carbono con una tarifa de 100 dólares por tonelada, en millones de dólares, 1988

\begin{tabular}{lrc}
\hline \multicolumn{1}{c}{ Países } & Recaudación & Porcentaje sobre el PNB (\%) \\
\hline OCDE-Europa & 1'098,000 & 1.99 \\
Francia & 121,000 & 1.28 \\
Alemania Occidental & 277,000 & 2.30 \\
Italia & 121,000 & 1.47 \\
Reino Unido & 188,000 & 2.31 \\
Canadá & 151,000 & 3.12 \\
Estados Unidos & 1'644,000 & 3.42 \\
Japón & 326,000 & 1.15 \\
OCDE-Total & 3'337,000 & 2.40 \\
\hline
\end{tabular}

Fuente: Poterba (1991).

yor sea la protección ambiental, menor será la capacidad recaudatoria del tributo en cuestión. Tal es la razón por la que deberían establecerse tributos lo suficientemente flexibles como para propiciar aumentos de sus tarifas o número de hechos gravados.

La recaudación de los tributos ambientales puede tener, básicamente, cuatro destinos diferentes:

a) La reducción del déficit público. Los ingresos obtenidos mediante un tributo ambiental podrían emplearse en el financiamiento de una reducción de la diferencia entre los gastos y los ingresos públicos, con la finalidad de conseguir cuentas públicas más saneadas.

b) Un incremento del gasto público. Otro de los usos alternativos de los ingresos de la imposición ambiental sería la financiación de un aumento del gasto público en, por ejemplo, las prestaciones por desempleo, las pensiones o, en suma, un mayor desarrollo del Estado de bienestar.

c) La reducción de otros impuestos. Con este empleo alternativo, basado en el principio de neutralidad fiscal, tratarían de conseguirse, en lo fundamental, dos objetivos: la mejora del ambiente y la implantación de determinadas políticas económicas. Tal opción favorecería, en concreto, la puesta en práctica del denominado doble dividendo, es decir, lograr una mejora del entorno natural y, al mismo tiempo, incrementar el empleo. 
d) Destino final de los ingresos. Este empleo de la recaudación obtenida mediante un tributo ecológico consiste, en esencia, en la financiación de determinadas actuaciones públicas ambientales. Ejemplo corriente de este destino de los ingresos ambientales es el que proporcionan las tasas fiscales ecológicas; los pagos realizados por este concepto tienen su razón de ser en la financiación de determinados servicios ambientales como, por ejemplo, las basuras locales.

Uno de los puntos de debate de la literatura disponible sobre fiscalidad ambiental es, en efecto, si la recaudación obtenida mediante las fuentes tributarias de esta naturaleza debería designarse a gastos ad hoc o, por el contrario, debería engrosar los ingresos del erario público. A continuación, siguiendo a Rozas Valdés (1997: 960-962), se destacan algunas ventajas e inconvenientes de la afectación de los ingresos de la tributación ecológica:

- Desde el ángulo de las ventajas de la afectación de la recaudación de los tributos ecológicos, dos son las más significativas: en primer lugar, es políticamente más sencillo justificar el establecimiento de esta clase de impuestos que el de otro tipo de tributos. La introducción de un impuesto ecológico tendría mayor aceptación en los contribuyentes, como consecuencia del aumento de la concientización de los ciudadanos respecto a la protección del medio. Sin embargo, si la introducción de una ecotasa no viniese acompañada de una reducción de otro(s) tributo(s), aumentaría la presión fiscal global. En segundo lugar, la administración pública que implantase el tributo ecológico aumentaría los recursos susceptibles de emplearse en la mejora del medio natural.

- En contrapartida, la afectación de la recaudación de los tributos ecológicos presentaría tres grandes inconvenientes: primero, la estructura del tributo quedaría sujeta a objetivos presupuestarios, lo que implicaría que la protección del entorno natural se regiría fundamentalmente por este tipo de criterios antes que por los exclusivamente técnicos; segundo, se dificulta la aplicación de políticas distributivas, ya que los ingresos fiscales ambientales no se utilizarían para disminuir otros tributos -en el marco del concepto de doble dividendo-, ni para aplicar políticas de compensación respecto a los grupos de referencia más afectados por la ecota- 
sa; y tercero, la dificultad para nivelar los ingresos y los gastos de las políticas ambientales haría que las actuaciones sobre el medio natural quedasen limitadas al nivel de recaudación de cada ejercicio presupuestario.

\section{Incidencia fiscal de los tributos ecológicos}

Los modelos empleados en el estudio de la incidencia impositiva de los tributos ambientales son, básicamente, los siguientes: de equilibrio parcial, de equilibrio general, input-output, de microsimulación y macroeconómicos

Centrándonos en el estudio de la incidencia impositiva de un tributo ambiental, éste debe realizarse en dos fases diferentes. En la primera se toman en cuenta cuatro alternativas de análisis, coherentes con otros tantos criterios: por una parte, que se valoren sólo los efectos directos o también los efectos indirectos, y, por otra, que se considere o no un cambio en el comportamiento de la demanda. La segunda fase consiste en ubicar cada una de estas cuatro alternativas en un ámbito temporal diferente -anual y vitalicio-. A su vez, cada una de las cuatro alternativas anteriormente señaladas puede considerarse como porcentaje o de la renta o gasto anual, o de la renta obtenida en el ciclo de vida familiar.

A la vista de estas dos fases y asumiendo las posibilidades reales de análisis, las principales vías para el estudio de la incidencia impositiva de los tributos ambientales se reducirían a dos:

a) Análisis de los efectos directos e indirectos considerando el cambio en el comportamiento de la demanda, sobre la base de los datos aportados por la renta obtenida o el gasto realizado anualmente por los grupos de referencia.

b) Análisis de los efectos directos e indirectos sin cambio de comportamiento de la demanda, sobre la base de la renta generada o el gasto realizado por los grupos de referencia en el año natural.

En el cuadro 2 se informa de modo esquemático sobre las posibles pautas de la traslación de un tributo ecológico que grave la contaminación atmosférica. En la columna 2 se indica cuál es el sujeto pasivo obligado al pago del impuesto; en las columnas 3 y 4 , quién podría ser el sujeto que realmente pagase el impuesto, y en las columnas 5 y 6 , quién debería ser el sujeto obligado a pagar el impuesto de acuerdo con el principio de "quien contamina paga". 


\section{Cuadro 2}

Alternativas para la traslación de un hipotético impuesto sobre las emisiones causantes de lluvia ácida

\begin{tabular}{|c|c|c|c|c|c|}
\hline \multirow[t]{2}{*}{ Energía } & \multirow{2}{*}{$\begin{array}{l}\text { Incidencia } \\
\text { inicial }\end{array}$} & \multicolumn{2}{|c|}{ Incidencia final } & \multicolumn{2}{|c|}{ ¿Quién debe pagar? } \\
\hline & & $\begin{array}{l}\text { Efectos } \\
\text { directos }\end{array}$ & $\begin{array}{c}\text { Efectos } \\
\text { indirectos }\end{array}$ & $\begin{array}{l}\text { Efectos } \\
\text { directos }\end{array}$ & $\begin{array}{l}\text { Efectos } \\
\text { indirectos }\end{array}$ \\
\hline $\begin{array}{l}\text { Energía } \\
\text { eléctrica }\end{array}$ & Productor & $\begin{array}{l}\text { - Productor } \\
\text { - Consumidor } \\
\text { final }\end{array}$ & $\begin{array}{l}\text { - Productor } \\
\text { - Consumidor } \\
\text { final } \\
\text { - Industria }\end{array}$ & Productor & Productor \\
\hline $\begin{array}{l}\text { Combustible } \\
\text { para el } \\
\text { transporte }\end{array}$ & Productor & $\begin{array}{l}\text { - Productor } \\
\text { - Consumidor } \\
\text { final }\end{array}$ & $\begin{array}{l}\text { - Productor } \\
\text { - Consumidor } \\
\text { final } \\
\text { - Empresas }\end{array}$ & $\begin{array}{l}\text { Consumidor } \\
\text { final }\end{array}$ & Empresas \\
\hline
\end{tabular}

Fuente: Elaboración propia.

\section{Conclusiones}

La importancia que ha adquirido el ambiente en las últimas décadas hace necesario el establecimiento de determinados instrumentos económicos, como los tributos ecológicos, que interioricen los efectos externos derivados de los procesos productivos. Sin embargo, estos instrumentos también ocasionarán impactos sobre determinadas magnitudes macroeconómicas y sobre las rentas de las familias. La importancia de los tributos ecológicos está llevando a su incardinación en los sistemas fiscales, y, para ello, deben arbitrarse también diversas pautas metodológicas para definir los efectos distributivos sobre determinados grupos de referencia. A lo largo de este trabajo hemos presentado una propuesta metodológica que, en nuestra opinión, debería valorarse en el momento de establecer o modificar un tributo ecológico, ya que una correcta distribución de la carga impositiva de los tributos ecológicos, con base en los principios de redistribución de la carga impositiva, es, sin lugar a dudas, sinónimo de éxito en la introducción de las ecotasas.

\section{Bibliografía}

Baker, Paul, Stephen Mekay y Elizabeth Symons (1990), The Simulation of Indirect Tax Reforms: SPIT, documento de trabajo W90/11, Insitute for Fiscal Studies, Reino Unido. 
Borrero Moro, Cristóbal (1999), La tributación ambiental en España, Tecnos, Madrid.

Brännlund, Runar y Bengt Kriström (1997), Energy and Environment Taxation in Sweden: Some Experience for the Swedish, documento presentado en el congreso internacional Environmental Implications of Market Based Policy Instruments, Suecia, noviembre.

Cronwell, Antonia y John Creedy (1996), “Carbon Taxation, Prices and Inequality in Australia", Fiscal Studies, Reino Unido, 17 (2): 21-38.

Hamilton, Kirk y Grant Cameron (1994): "Simulating the Distributional Effects of a Canadian Carbon Tax", Canadian Public Policy, Canadá, xx (4): 385-399.

Johnson, Paul, Stephen McKay y Spend Smith (1990), The Distributional Consequences of Environmental Taxes, Institute for Fiscal Study, Reino Unido.

López-Guzmán Guzmán, Tomás J. (2002), Fiscalidad ambiental: análisis y efectos distributivos, Comares, España.

OCDE (Organización para la Cooperación y el Desarrollo Económicos) (1994), The Distributive Effects of Economic Instruments for Environmental Policy, OECD Documents, Francia.

Pearson, Mark (1995), “The Political Economy of Implementing Enviornmental Taxes", International Tax and Public Finance, Reino Unido, 2 (2): 357-373.

Poterba, James M. (1991), “Tax Policy to Combat Global Warming: On Designing a Carbon Tax", en Rudigero Dornbusch y James M. Poterba (eds.), Global Warming: Economic Policy Responses, MIT Press, Massachusetts, pp. 47-67.

Rozas Valdés, José Andrés (1997) "El impuesto gallego sobre la contaminación atmosférica", Revista de Derecho Financiero y Hacienda Pública, España, XLVII (246): 943-968. 
Smith, Stephen (1992), Distribution Effects of European Carbon Tax, documento de trabajo 22.92, Fondazione Eni Enrico Mattei, Italia.

Symons, Elizabeth, John Proops y Philip Gay (1994), “Carbon Taxes, Consumer Demand and Carbon Dioxide Emissions: A Simulation Analysis for the UK", Fiscal Studies, 15 (2): 19-43.

Symons, Elizabeth, Stefan Speck y John R. Proops (2004), The Distributional Effects of European Pollution and Energy Taxes (en prensa).

Whalley, John y Randall Wigle (1991), “The International Incidence of Carbon Taxes", en Rudigero Dornbusch y Janes M. Poterba (eds.) Global Warming: Economic Policy Responses, MIT Press, Massachusetts, pp. 233-263.

Recibido: 30 de agosto de 2004. Reenviado: 20 de junio de 2005. Aceptado: 15 de julio de 2005.

Tomás J. López-Guzmán Guzmán es doctor en ciencias económicas y empresariales. Labora en el Área de Economía Aplicada, Facultad de Ciencias del Trabajo, de la Universidad de Córdoba (España). Como líneas de investigación maneja economía ambiental e impacto económico del sector turístico. Entre sus publicaciones están: "Características diferenciales de la financiación local de las ciudades con estatuto de autonomía", Revista de Estudios Regionales, núm. 66, pp. 251-259, 2003; "La aplicación de los permisos de emisión negociables en Dinamarca. Un ejemplo para España”, Boletín Económico de ICE, núm. 2800, pp. 4755, 2004; "Environmental Taxes in Spain", documento de trabajo 5/04, Roskilde University, Dinamarca, 2004, y "El turismo como motor de desarrollo económico en ciudades patrimonio de la humanidad", Pasos, revista de Turismo y Patrimonio Cultural, 2 (2): 243-256, 2004.

Fernando Lara de Vicente es licenciado en ciencias económicas y empresariales. Trabaja en el Área de Economía Aplicada de la Facultad de Ciencias del Trabajo en la Universidad de Córdoba 
(España). Líneas actuales de investigación: economía ambiental y turismo sostenible. Publicaciones: con otros, Observatorio económico de la provincia de Córdoba 1998, Diputación de Córdoba, 2000; "El gasto público en el contexto de la Unión Económica y Monetaria”, en José Manuel Cansino (coord.), Evaluación de politicas públicas, Universidad de Sevilla-Fundación Valcel, pp. 47-63, 2003; "La aplicación de los permisos de emisión negociables en Dinamarca. Un ejemplo para España”, Boletín Económico de ICE, núm. 2800, pp. 47-55, 2004, y "El turismo como motor de desarrollo económico en ciudades patrimonio de la humanidad", Pasos, revista de Turismo y Patrimonio Cultural, 2 (2): 243-256, 2004. 